\title{
14
}

\section{New Approaches for Collaborative Learning and Group Activities}

\author{
A. Paiva \\ INESC and IST, \\ Rua Alves Redol, 9, 1000 Lisboa, Portugal \\ e-mail:Ana.Paiva@inesc.pt
}

\begin{abstract}
One of the most promising topics of study and exploration for the last decade in computer based learning has been collaborative learning. However, it is only recently that the approaches explored are being tested and effectively used. This brief summary focuses on the problems and new approaches of collaborative learning, as highlighted by the discussions held during the collaborative learning session at the IFIP $3.3 \& 3.6$ Joint Conference.

Keywords

Computer supported collaborative learning, Collaborative learning, Active support, Joint space, Interaction, Coordination
\end{abstract}




\section{INTRODUCTION}

The last decade has seen not only the impressive development of computer based collaborative learning, but also the exponential emergence of Web based tools. So, it is not surprising that the new approaches in this area are centred on the use of Web based technology. Indeed, the emphasis has shifted on learning as a constructive process where the learners are emerged in a social environment for task execution. Furthermore, this process has crossed the borders of the small classes to embrace a world wide scenario where collaboration at a distance is now a reality. One can predict that in the "Virtual Campuses" of the next decade, now starting to be built, collaborative learning and Web based distance learning will play important roles.

However, although the telecommunications infrastructure supporting the collaboration at a distance has had an almost explosive development, it is also true that these technological facilities do not replace the need for contextual and task oriented support. This kind of rich support was a common theme in all the presentations and discussions held in the session of "Collaborative Learning" of the IFIP 3.3 and 3.6 Joint Working Conference. This paper summarises their finding with a focus on distance learning systems which "support collaborative critical discussions at a distance" with the goal to promote learning. The present document is structured as follows: first a small overview of tools for collaborative learning is made. Then, some of the aspects found and reported in the Conference are highlighted, and the intriguing results obtained are discussed. Finally, some questions that were raised at the discussion are presented, leaving them open as new problems to be addressed.

\section{SUPPORTING COLLABORATIVE DISCOURSE AT A DISTANCE}

According to the socio-constructivist (see (Dillenbourg et al. 1997)) approach to learning, it is through the interaction with peers, and by comparing one's views with those of the others, that learners start to understand new approaches to reality (see (Doise, 1990)). The presence of learning companions with whom the students can interact, change information and share task execution, gives to the learning process a more real social context, and thus provides more effective environments for learning.

During the last decade, several environments have been proposed in order to support these collaborative situations. Results were obtained in classes of students exchanging email as well as in using collaborative virtual environments. In fact, the tools that support collaborative learning have changed considerably during the last few years, and at present, they range from the general purpose environments (that support email, talk, chat, etc.) to the more specific ones, tied to one task in 
hand. In general, they intend to support some of the mechanisms that are observed in collaborative learning scenarios such as (see (Dillenbourg, 1995)): (1) conflict (socio-cognitive conflict), (2) mutual and auto-explanation, (3) internalisation, (4) appropriation of the knowledge and tasks of the peers, (5) mutual regulation, and (6) social grounding.

However, from the present state of research, it is not clear which approach should be adopted for building a collaborative learning tool, nor which type of support should be given in order to support some of these mechanisms. Such support can range from a very basic one with only communication infrastructure mechanisms to highly task oriented ones. In general, one can divide the support given by collaborative learning tools in three main types: social support, task support and cognitive support.

Social support: A tool must promote the emergence of social activities, including conflicts between peers, helping learners to achieve a shared understanding. This type of support includes support for information and knowledge share.

Task support: A tool must promote task level execution and its coordination. This includes the support of a shared working space, in which the students can construct in their activities.

Cognitive Support: the tool must promote cognitive and meta-cognitive episodes, such as reading, understanding, planning and analysing. They can support the construction of mutual explanations between students, or promote individual internalisation.

Taking a different perspective, one can also divide the support given to collaborating students into two main classes: active support and passive support.

Passive support is given by a tool in the form of implicit imposed restrictions on the ways learners interact, collaborate, solve problems and think. For example, both the work by (Barros, et al. 1998) and (Hietala et al. 1998) describe tools which include passive support by embedding in the interface a set of pre-defined collaborative interactions.

Active support is provided by an external element that helps the learners to overcome difficulties in the collaboration process. This active role of support can be played by a teacher or even by the tool itself (for example, in the Quorum project (Canas et al. 1995) the real teacher plays a role of a mediator).

\subsection{Collaborative Tasks}

The nature of the task (e.g. whether it is shareable or not) influences the results of the collaboration (see (Dillenbourg 1997) for a discussion) as well as the whole process. This influence is well illustrated by the papers (Barros et al. 1998) and (Hietala et al. 1998).

The intriguing findings obtained by (Hietala et al. 1998) demonstrate that even using the same collaborative learning environment, different results can be obtained for different tasks. Since the tasks can be set up by the teacher, several topics were explored and better results were achieved if the task at hand depends 
significantly on achieving a common objective. Similarly, the task to be executed by the learners described in (Barros et al. 97) was writing a summary, which clearly establishes an objective to be achieved. This required mastering two types of skills. First, a content based one, where the students had to relate aspects of a certain subject and create a summary of that subject, and second, students had to learn how to work in a cooperative manner, which included skills such as being able to propose or explain ideas. In both of these studies there is a clear need for supporting the process of collaboration towards achieving a common objective.

\subsection{Joint Problem Space}

Participation in a joint problem solving process is a way to foster the understanding of the problem, where each partner, in the collaboration process, changes his views by giving meaning to the others actions according to his or her own conceptual framework. Indeed, one essential property of collaborative problem solving is that it enables the construction of a shared conceptual structure that is called Joint Problem Space (introduced by (Teasley \& Rochelle 1993)). The Joint Problem Space is intended to support the collaborative activities by integrating semantic interpretations of the goals, features, operators ad methods.

Both the work by (Barros et al. 1998) and (Hietala et al. 1998) illustrate the creation of a joint problem space.

\subsection{Types of Interactions}

The achievements of the collaboration not only depend on the task but also on the types of interactions. The interactions required in the collaboration process may prompt learners to express beliefs in ways that serve to organise what they know and identify gaps in their understanding. However, some interactions have a more positive influence in the learning results than others. So, which kind of interactions can be identified as collaborative? And how do systems trigger such interactions?

For example, the Collaboratory Notebook (see (Edelson et al. 1996)) provides shared hypermedia databases that the learners construct based on specific scientific inquiry forms of interactions. The information exchanged and stored by the students through questions, conjectures, evidence for and against conjectures, plans, steps in plans, information and commentary. In the shared workspace the students make links from each one of these components of the inquiry model.

Indeed, as mentioned in (Hietala et al. 1998) an important stage of learning (called dialogue) shapes and re-organises the material learned through discussions, mutual questioning and reflection in a social environment. However, the main problem lies in the fact that students need appropriate support in order to be assisted in the dialogue phase.

One solution to this problem is offered by (Barros et al. 1998) and (Edelson et al. 1996) through the use of a restricted set of pre-defined interaction types allowed between the learners. In the passive support introduced by (Barros et al. 1998) the interactions are task oriented and structured by the use of proposals, contra- 
proposals, questions and comments. These collaborative dialogue acts, support the students in following a pattern of communication such that they are forced to justify their actions.

Field studies results with such systems indicate that supporting collaboration through structured and restricted dialogue acts lead to better results, both at University level (in the case of (Barros et al. 1998) and (Hietala 1998)) and school children (see (Baker \& Lund 1996)).

\subsection{Coordination Spaces}

Finally, in order to support the concurrent execution of activities by the learners, some of these tools provide a coordination space. This coordination varies from tool to tool, which in general rely on "versions" and "voting" principles. The final result may pass through different versions demarcating different stages of the work (e.g. from the stage of "proposed" into the "final" stage (Barros et al. 1997)).

\section{SOME RESULTS AND DISCUSSION}

It is interesting to observe the convergence of the results presented by (Barros et al. 1998) and (Hietala 1998) with those found previously by (Baker \& Lund 1996). A common theme is the provision of passive support to collaborative learning through the use of "structured" collaborative interactions. This is usually embedded in the interface in a way that forces the learners to follow a pattern of collaboration. However, the findings also suggest that such structuring is not enough to captivate the students that are unable to play active roles in collaborative tasks. Students may even avoid using these very structured tools, and prefer a more free style of interaction. Since the motivation factor is indeed important to obtain good results is there a need for the students to introduce their own "communication acts"? This question remains to be explored in the future.

On the other hand, (Hietala 1998) showed that the more positive results were achieved when the students where "obliged" to use the system. So, how can these tools motivate students that do not participate? In trying to find answers to such question it was suggested that the motivation factor can come from an "elected student", a "teacher", or some "computer based mediator". However, such a solution raises other questions such as what is the impact on the role of the teacher resulting from the use of computer based collaborative learning tools.

\section{REFERENCES}

Baker, M. \& Lund. K. (1996) Flexibly structuring the interaction in a CSCL environment, in European Conference on Artificial Intelligence in Education (eds. P. Brna, A. Paiva \& J. Self), Edicoes Colibri, ISBN 972-8288-37-9. 
Barros, B., Rodriguez-Artacho, A. \& Verdejo, F. (1998) Towards a Model of Collaborative Support for Distance Learners to Perform Joint Tasks, in The Virtual Campus: Trends for Higher Education and Training, (eds. F. Verdejo $\&$ G. Davies) Chapman \& Hall (in this volume).

Canas, A., Ford, K., Hayes, P., Brennan J. \& Reichherzer T.(1995) Knowledge Construction and Sharing in Quorum, in Proceedings of the AIED'95, (ed. J. Greer), AACE..

Dillenbourg, P., Baker, M., Blaye, A. \& O'Malley, C. (1997) The evolution of research on collaborative learning, in Collaborative Learning, (ed. C. O’Malley) Springer Verlag, 1997.

Doyse, W. (1990) The development of individual competencies through social interaction, in Children helping children (eds. H.Foot, M.Morgan \& R. Shute) J.Wiley \& sons..

Edelson, D., Pea, R. \& Gomez, L. (1996) The Collaboratory Notebook, Communications of ACM 39(4), April.

Hietala, P., Niemirepo, T. \& Ovaska, S. (1998) Using the World Wide Web to promote educational discussion on university level courses, in The Viriual Campus: Trends for Higher Education and Training, (eds. F. Verdejo \& G. Davies) Chapman \& Hall (in this volume).

S.Teasley, S. \& Rochelle, J. (1993) Constructing a Joint Problem Space: The Computer as a Tool for Sharing Knowledge, in Computers as Cognitive Tools, (ed. S. Lajoie \& S. Derry), Lawrence Erlbaum Associates.

\section{BIOGRAPHY}

Ana Paiva is a Professor of Computer Science at IST, Technical University of Lisbon and a Research Fellow at INESC. She completed her $\mathrm{PhD}$ in 1996 in the University of Lancaster, UK, in the area of Learner Modelling for Interactive Learning Environments. Since then she has been working on Learner Modelling, Pedagogical Agents and Collaborative Learning. She participated in many international conferences and was one of the co-chairs of the 1996 European Conference on Artificial Intelligence in Education held in Lisbon.

Currently, she is co-ordinating several Computer Based Learning projects in collaboration with Universities, industry and schools. She also lectures on Computer Science undergraduate courses and is responsible for MSc courses on Computer Based Learning and Intelligent Agents. 